\title{
Hemangioma epididimario trombosado en paciente mayor. Presentación de 1 caso con revisión conceptual y de la literatura
}

\author{
Ruiz Liso JM*, Ruiz García J*, Pardo López Mán*, Vaillo Vinagre A*, Gutiérrez Martín A*, \\ Bermúdez Villaverde $\mathrm{R}^{* *}$.
}

*Servicio de Patología. Complejo Hospitalario de Soria (CHS). Hospital Virgen del Mirón. Soria. **Servicio de Urología. Hospital Gregorio Marañón. Madrid.

Actas Urol Esp. 2008; 32(5):533-541

\section{RESUMEN}

\section{HEMANGIOMA EPIDIDIMARIO TROMBOSADO EN PACIENTE MAYOR. PRESENTACIÓN DE 1 CASO CON REVISIÓN CONCEPTUAL Y DE LA LITERATURA.}

Introducción: Aunque teóricamente en la región paratesticular puede desarrollarse cualquier tipo de tumor benigno de tejidos blandos, son los lipomas, leiomiomas y tumores adenomatoides los más frecuentes, mientras que los hemangiomas son menos comunes. Aportamos un nuevo caso de hemangioma intraescrotal (H I), localizado en epidídimo, valorando la bibliografía que nos permita conocer la incidencia y características clínicopatológicas de estos tumores.

Material y métodos: Se incluye para estudio un paciente de 74 años con tumoración escrotal derecha, a quien se practica orquiectomía. Se realiza estudio histopatológico. De igual forma se realiza un examen bibliográfico de este tipo de tumores a través de Medline y de búsqueda no indexada con revisión conceptual y de la literatura.

Resultados: Se corresponde la lesión con un HI cavernoso trombosado de epidídimo con parénquima testicular respetado. Su diagnóstico histopatológico no presenta problemas, mientras que el clínico y ultrasonográfico, alcanza todo el espectro de tumores de partes blandas, benignos y malignos. El número de hemangiomas intraescrotales epididimarios descritos en la literatura es muy reducido.

Conclusiones: Los tumores escrotales, precisan de un estudio preoperatorio clínico y ecográfico completo, a pesar de que la mayoria son diagnosticados tras su extirpación quirúrgica. El complejo sistema tisular de la zona paratesticular implica que el diagnóstico diferencial incluso no permita diferenciar tumores malignos de benignos. La baja incidencia de muchas de estas neoplasias ha condicionado la falta de protocolos estandarizados para su diagnóstico pero también para su tratamiento unificado.

Palabras clave: Hemangioma. Epidídimo. Tumor. Escrotal. Cavernoso.

\section{ABSTRACT \\ EPIDIDYMIS TROMBOSED HEMANGIOMA IN OLD PATIENT. PRESENTATION OF 1 CASE WITH CONCEPTUAL REVIEW AND OF THE LITERATURE}

Introduction: Though theoretically in the paratesticular region one can develop any type of benign tumour of soft tissues, they are the lipomas, leiomyomas and adenomatoid tumours the most usual whereas the hemangiomas are less common. We contribute a new case of epididymis intrascrotal hemangioma (H I), valuing the bibliography that allows us to know the incidence and clinic-pathologic characteristics of these tumours.

Material and Metod: A 74-year-old patient includes for study with right scrotal tumour, who is practised orchiectomy. Hystopathologic study is realized. Of equal form there is realized a bibliographical examination of this type of tumours across Medline and of not index-linked search with conceptual review and of the literature.

Results: The injury corresponds with a HI cavernous trombosed of epididymis with testicular parenchyma respected. Your hystopathologic diagnosis does not present problems, whereas the clinical one and ultrasonographic study, reaches the whole spectrum of tumours, benign and malignant of soft tissues. The number of intrascrotal hemangiomas of epididymis described in the literature is very limited.

Conclusions: The scrotal tumours are necessary a preoperatory clinical and sonographic study completely, in spite of the fact that they are diagnosed the majority after your surgical removal. The complex system of the tissues on the zone paratesticular implies that the differential enclosed diagnosis does not allow to differentiate, malignant tumours of benign. The low incident of many of these masses has determined the mistake of standardized protocols for your diagnosis but also for their unified treatment.

Keywords: Hemangioma. Epididymis. Tumour. Scrotal. Cavernous. 
$\mathrm{L}^{a}$ a anatomía intra-escrotal es compleja. En su interior contiene como estructuras fundamentales, además de los testículos, el conducto deferente, las túnicas vaginales testiculares, el epidídimo y restos vestigiales. Histogenéticamente, esta zona está compuesta de varios tejidos: epiteliales, mesoteliales y mesodérmicos. Por esta razón, las estirpes tumorales que surgen en esta región son heterogéneas y con diferente patobiología. Clínicamente, los tumores paratesticulares son indistinguibles de los tumores testiculares, lo que provoca que inicialmente se presten a un diagnóstico, en muchos casos, erróneo. La mayoría se presentan como una masa escrotal o bien como una tumefacción que puede ser o no dolorosa y ocasionalmente acompañarse de un hidrocele. No suele distinguirse preoperatoriamente entre lesiones benignas y malignas paratesticulares, lo que incide en un problema sobreañadido ${ }^{1-3}$.

Los tumores escrotales y en concreto los paratesticulares son médica y bibliográficamente escasos y como tales, es difícil conocer su verdadera incidencia, la cual no ha sido nunca establecida con objetividad $^{1-5}$.

El primer hemangioma intraescrotal (HI) con afectación del epidídimo, lo describe Robertson en el año $1954^{6}$, aunque el primer hemangioma escrotal corresponde a una publicación de Boullay en $1851^{7}$.

Siendo infrecuentes, los tumores paratesticulares tienen una significativa tasa de benignidad, próxima al 70\%1,2. Este porcentaje, probablemente, es más alto si solo consideramos los varones adultos. Señalar, también, que la incidencia de las lesiones paratesticulares de partes blandas es difícil de estimar, especialmente en el caso de los tumores benignos, ya que por lo general no suelen ser publicados $^{5}$. Lo que es evidente es que la mayoría de los tumores malignos paratesticulares son sarcomas. Significar, también, que la presencia de hidrocele en asociación con un tumor paratesticular se ha asociado en muchas ocasiones como un signo de malignidad $^{2,3,8-12}$ y ello ha conducido a diagnósticos preoperatorios no concordantes con la biología neoplásica.

Dentro de los tumores anexiales benignos, el tumor adenomatoide de epididimo representa un alto porcentaje de los tumores paratesticulares, con ecogenicidad variable y localización preferente en cola de epidídimo (más del 50\%). El pseudotumor fibroso, y el lipoma del cordón espermático seguirían en frecuencia al primero. Indicar también que el cistadenoma de epidídimo, siendo una lesión rara y la única de estirpe epitelial, es bilateral en un 34\% de los casos, observándose con cierta frecuencia asociado a la enfermedad de Von Hippel-Lindau ${ }^{8-13}$.

En general, los tumores benignos son homogéneos e hiperecoicos, mientras que los malignos son o hipoecoicos de forma homogénea o bien tienen un patrón heterogéneo con áreas hipo e hiperecoicas. No obstante, se han visto liposarcomas hiperecoicos y homogéneos, por lo que la ultrasonografía no es concluyente en un gran número de $\operatorname{casos}^{8-11}$.

Al mismo tiempo, debemos separar claramente los tumores localizados en la piel y el tejido celular subcutáneo de la bolsa escrotal, de los verdaderos tumores del escroto, o intraescrotales. En muchos casos de los publicados hasta ahora, y en concreto, de los tumores vasculares benignos, no se identifica claramente su localización, que queda manifestada como hemangioma escrotal, sin especificar localización precisa. Al no tener ninguna correlación entre las estructuras cito e histogenéticas de procedencia, ni tampoco en su incidencia -muy inferior en los intraescrotales-, es importante su división y su estudio independiente.

En este artículo revisamos las características clínicopatológicas de los hemangiomas intraescrotales (HI) paratesticulares, publicados hasta la fecha, aportando un nuevo caso con un patrón anatomopatológico y etario, infrecuente.

A pesar de todo, y como patrones diagnósticos diferenciales a valorar en esta localización, debemos utilizar la última clasificación de la O.M.S. de los tumores mesenquimales del escroto, cordón espermático y anejos testiculares realizada en $2004^{2}$ que incluye:
- Lipoma
- Linfangioma
- Hemangioma
- Leiomioma
- Neurofibroma
- Schwannoma
- Tumor de células granulares
- Angiofibroma celular: tumor tipo angiomiofi- broblastoma del varón
- Pseudotumor fibroso calcificante
- Hamartoma fibroso de la Infancia
- Liposarcoma 
- Leiomiosarcoma

- Fibrohistiocitoma Maligno

- Fibrosarcoma

- Rabdomiosarcoma

A ellos deberíamos añadir el Tumor desmoplásico de células redondas ${ }^{2,3}$.

$\mathrm{Y}$ valorar en los diagnósticos, el conjunto de tumores benignos descritos en estas áreas, que incluimos en la Tabla 1.

Considerar por último que las masas intratesticulares, sólidas, deben considerarse preoperatoriamente malignas en principio y benignas las extra/ paratesticulares escrotales.

\section{MATERIAL Y MÉTODO}

\section{Revisión de la Literatura (Tabla 2)}

Los HI son tumores muy raros. Revisada la literatura científica a través de Medline y búsqueda no indexada, hemos encontrado publicados hasta el mes de Septiembre de 2007 un total -tan solode 10 hemangiomas de epidídimo y de ellos, tan solo 4 cavernosos -todos en adultos-, siendo el nuestro el de mayor edad. En las áreas paratesticulares, hemos hallado un total de $140 \mathrm{HI}$, de los que carecemos de localización objetiva escrotal (sd) y lateralidad en 54 casos. En otros 19 conocemos su lateralidad (izquierda o derecha) pero no su localización intra o paratesticular, que es confusa.

Nuestro caso es, pues, el número 141 de los HI en las series, tras su examen y reevaluación histológica contrastada. Los subtipos histológicos presentan también un amplio espectro que, en una revisión actualizada, si observáramos las preparaciones histológicas, probablemente habría cambios en su panel diagnóstico.

En 58 casos de HI conocemos su edad al diagnóstico. La edad media -global- de presentación de los HI es de 29,2 años, con un intervalo etario desde "feto" 36 a 75 años -un caso de túnica albugínea-; aunque, si separamos en grupos etarios los HI, obtenemos medias -edades- que nos aproximan más a la realidad. Así, es 50,1 años, la edad media de los 28 casos recogidos (con edad incluida) entre 21-75 años, y 8,94 años los 30 casos en menores de 20. De estos últimos, es 5,24 años entre los 19 casos menores de 10 años y 16,5 años en el grupo etario de 11 a 20 años.

Tabla 1. Lesiones benignas escrotales (no subcutáneas) Ruiz Liso et al."

\begin{tabular}{|c|c|c|c|c|c|c|}
\hline \multicolumn{7}{|l|}{ FRECUENTES } \\
\hline $\begin{array}{l}\text { Tumor } \\
\text { adenomatoide }\end{array}$ & $\begin{array}{l}\text { Lipoma y } \\
\text { variantes: } \\
\text { angio, leio y } \\
\text { mixolipoma }\end{array}$ & $\begin{array}{l}\text { Hiperplasia } \\
\text { adenomatosa } \\
\text { de epidídimo }\end{array}$ & Leiomioma & $\begin{array}{l}\text { Granuloma } \\
\text { espermático }\end{array}$ & $\begin{array}{l}\text { Quiste } \\
\text { epididimario }\end{array}$ & Varicocele \\
\hline Espermatocele & Hidrocele & $\begin{array}{l}\text { Vasitis } \\
\text { nodosa }\end{array}$ & $\begin{array}{l}\text { Quiste } \\
\text { mesotelial }\end{array}$ & $\begin{array}{l}\text { Pseudotumor } \\
\text { inflamatorio }\end{array}$ & $\begin{array}{l}\text { Hiperplasia } \\
\text { mesotelial }\end{array}$ & $\begin{array}{l}\text { Quistes dermoides } \\
\text { del cordón espermático }\end{array}$ \\
\hline \multicolumn{7}{|c|}{ POCO FRECUENTES } \\
\hline Hemangioma & Linfangioma & Schwanoma & Angiofibroma & Fibroma & $\begin{array}{l}\text { Mesotelioma } \\
\text { fibroso }\end{array}$ & $\begin{array}{l}\text { Cistadenoma } \\
\text { seroso paratesticular }\end{array}$ \\
\hline Cistadenoma & Tumor desmoide & Neurofibroma & $\begin{array}{l}\text { Mesotelioma } \\
\text { papilar }\end{array}$ & $\begin{array}{l}\text { Mesotelioma } \\
\text { quístico } \\
\text { benigno }\end{array}$ & $\begin{array}{l}\text { Granuloma } \\
\text { de colesterol }\end{array}$ & $\begin{array}{l}\text { Hamartoma fibroso } \\
\text { de la infancia }\end{array}$ \\
\hline \multicolumn{7}{|c|}{ CASOS AISLADOS } \\
\hline $\begin{array}{l}\text { Restos } \\
\text { adrenales }\end{array}$ & $\begin{array}{l}\text { Periorquitis } \\
\text { meconial }\end{array}$ & $\begin{array}{l}\text { Teratoma } \\
\text { de predominio } \\
\text { mesodérmico }\end{array}$ & $\begin{array}{l}\text { Angiomio- } \\
\text { fibroblastoma }\end{array}$ & $\begin{array}{l}\text { Proliferación } \\
\text { nodular } \\
\text { y fibrosa de } \\
\text { epidídimo }\end{array}$ & Paraganglioma & $\begin{array}{l}\text { Granuloma } \\
\text { inflamatorio } \\
\text { calcificante de la } \\
\text { rete testis }\end{array}$ \\
\hline $\begin{array}{l}\text { Lipogranuloma } \\
\text { esclerosante }\end{array}$ & $\begin{array}{l}\text { Angiomixoma } \\
\text { superficial y } \\
\text { agresivo }\end{array}$ & Rabdomioma & Perineurioma & $\begin{array}{l}\text { Tumor de } \\
\text { células } \\
\text { granulares }\end{array}$ & $\begin{array}{l}\text { Miofibroblastoma } \\
\text { de túnicas } \\
\text { testiculares }\end{array}$ & \\
\hline
\end{tabular}

(*)Elaboración personal por los autores ${ }^{14-92}$ 
Tabla 2. Localización de los tumores vasculares testiculares y paratesticulares(*)

\begin{tabular}{|c|c|c|c|c|c|c|c|c|c|c|c|c|c|c|c|c|c|c|}
\hline \multirow{2}{*}{$\begin{array}{l}\text { Hemangiomas } \\
\text { Localización }\end{array}$} & \multicolumn{3}{|c|}{$\begin{array}{l}\text { Escrotal sin } \\
\text { datos }\end{array}$} & \multicolumn{3}{|c|}{ Testiculo } & \multicolumn{3}{|c|}{$\begin{array}{l}\text { Túnica } \\
\text { albuginea }\end{array}$} & \multicolumn{3}{|c|}{ Epidídimo } & \multicolumn{3}{|c|}{$\begin{array}{l}\text { Cordón } \\
\text { espermático }\end{array}$} & \multicolumn{3}{|c|}{ Total } \\
\hline & Iz & $\mathrm{Sd}$ & $\mathrm{Dh}$ & Iz & $\mathrm{Sd}$ & $\mathrm{Dh}$ & $\mathrm{Iz}$ & $\mathrm{Sd}$ & $\mathrm{Dh}$ & $\mathrm{Iz}$ & Sd & $\mathrm{Dh}$ & $\mathrm{Iz}$ & $\mathrm{Sd}$ & $\mathrm{Dh}$ & Iz & $\mathrm{Sd}$ & $\mathrm{Dh}$ \\
\hline Cavernoso & 2 & 0 & 1 & 5 & 8 & 4 & 2 & & 2 & 2 & & 2 & 4 & & 1 & 15 & 8 & 10 \\
\hline Capilar & 1 & 0 & 0 & 2 & 4 & 2 & 0 & 1 & 0 & 2 & & 0 & 0 & & 1 & 3 & 5 & 3 \\
\hline Hemangioendotelioma & 2 & 0 & 1 & 0 & & 1 & 0 & & 0 & 0 & & 0 & 0 & & 0 & 2 & 0 & 2 \\
\hline Venoso & 1 & 0 & 1 & 1 & & 0 & 0 & & 0 & 0 & & 0 & 0 & & 0 & 2 & 0 & 1 \\
\hline Esclerosante & 0 & 0 & 0 & 0 & & 0 & 0 & & 0 & 0 & & 1 & 0 & & 0 & 0 & 0 & 1 \\
\hline Hemangiolinfangioma & 1 & 0 & 2 & 3 & & 1 & 0 & & 0 & 0 & & 0 & 0 & & 0 & 4 & 0 & 3 \\
\hline Histiocitoide & 1 & 0 & 0 & 0 & 5 & 0 & 1 & & 0 & 0 & 3 & 0 & 0 & & 0 & 2 & 8 & 0 \\
\hline Hemangiofibroleioma & 1 & 0 & 0 & 0 & & 0 & 0 & & 0 & 0 & & 1 & 0 & & 0 & 1 & 0 & 1 \\
\hline Arteriovenoso & 0 & 0 & 0 & 1 & & 0 & 0 & & 0 & 0 & & 0 & 0 & & 0 & 1 & 0 & 0 \\
\hline Fibroangiomioma & 1 & 0 & 1 & 1 & & 0 & 0 & & 0 & 0 & & 0 & 0 & & 0 & 2 & 0 & 1 \\
\hline Angiofibroblastoma & 2 & 0 & 0 & 0 & & 0 & 0 & & 0 & 0 & & 0 & 0 & & 0 & 2 & 0 & 0 \\
\hline Terebrante & 1 & 0 & 0 & 0 & & 0 & 0 & & 0 & 0 & & 0 & 0 & & 0 & 1 & 0 & 0 \\
\hline Otros Sin Datos & & 54 & & & 2 & & & 1 & & & 1 & & & 5 & & & 63 & \\
\hline
\end{tabular}

Angiomas Sd

Angiomixoma,

Angiofibroma,

Angiomio-

Fibroblastoma,

Etc...

$\begin{array}{lllllllllllllllllllllllll}\text { Totales } & 13 & 54 & 6 & 13 & 19 & 8 & 3 & 2 & 2 & 2 & 4 & 4 & 4 & 5 & 2 & 35 & 84 & 22\end{array}$

(*)Elaboración personal de la revisión por los autores ${ }^{18-92}$

De los 58, solo reflejan manifestación de dolor por la tumoración o a la exploración 19 casos, de ellos la mayoría cavernosos (dato, solo consignado en 27 casos).

La presencia de trombos, en principio esperados, preferentemente en los cavernosos, tan solo la hemos encontrado registrada específicamente, en tres casos de HI con infarto testicular asociado, en un caso localizado en túnica vaginal y en otro de cordón espermático, sin quedar reflejado este hecho en las descripciones de la mayoría de las revisiones. Nuestro caso se suma a los anteriores.

La lateralidad de los hemangiomas escrotales es discretamente mayor en el lado derecho, de forma global y en los HI cavernosos.

De los 141 casos de HI, 57 corresponden a autores y publicaciones japonesas, que son en el campo de la urología oncológica, los más fecundos.

\section{Revisión conceptual}

Durante muchos años, el HI se ha valorado como un tumor más frecuente en niños. Sin embargo, las cifras encontradas en la revisión realizada no muestran prácticamente diferencias.
En los adultos, los HI se presentan como masas escrotales nodulares discretas, frecuentemente próximas al parénquima testicular o surgiendo de él (intratesticulares) así como en menor número del cordón espermático y menos a menudo en el epidídimo, con neta separación del parénquima testicular. Son palpables en el canal inguinal o/y en el escroto, y antes de cualquier intervención quirúrgica deberían ser evaluadas por ecografía escrotal.

En el caso de los hemangiomas cavernosos del escroto, la ecografía presenta un problema de heterogeneidad sonográfica confusa que debe ser contrastada y evaluada en todos los casos de sospecha. El dolor no es un signo objetivo, asociándose más a compromisos vasculares que a los propios tumorales, salvo en grandes masas.

La orquiectomía -por la afectación de estructuras vecinas anexiales- suele ser el tratamiento de la mayoría de los HI con compromiso orgánico.

Los datos histológicos son escasos en una gran parte de las publicaciones y ausentes en muchas de las series revisadas.

Si la biopsia intraoperatoria confirma el origen sarcomatoso o maligno de la lesión, procede realizar 
una orquiectomía radical con ligadura alta del cordón espermático y escisión de todos los tejidos blandos periféricos.

\section{CASO CLÍNICO}

Paciente varón de 74 años, sin antecedentes de interés (glaucoma e HTA) con tumoración escrotal derecha indolora localizada a la palpación en epidídimo derecho, sin alteraciones en el izquierdo. Masa dura, irregular de consistencia pétrea que va de cabeza a cola de epidídimo derecho. Parénquima testicular derecho normal. Hemiescroto izquierdo normal. No fiebre ni síndrome miccional. Ausencia de adenopatías inguinales. Analítica: alfa fetoproteína y beta HCG en rangos normales así como el PSA.

Ecografia: Masa heterogénea en cordón espermático y cabeza de epidídimo de testículo derecho. con zonas hipo e hiperecoicas. Resto normal (Fig. 1).

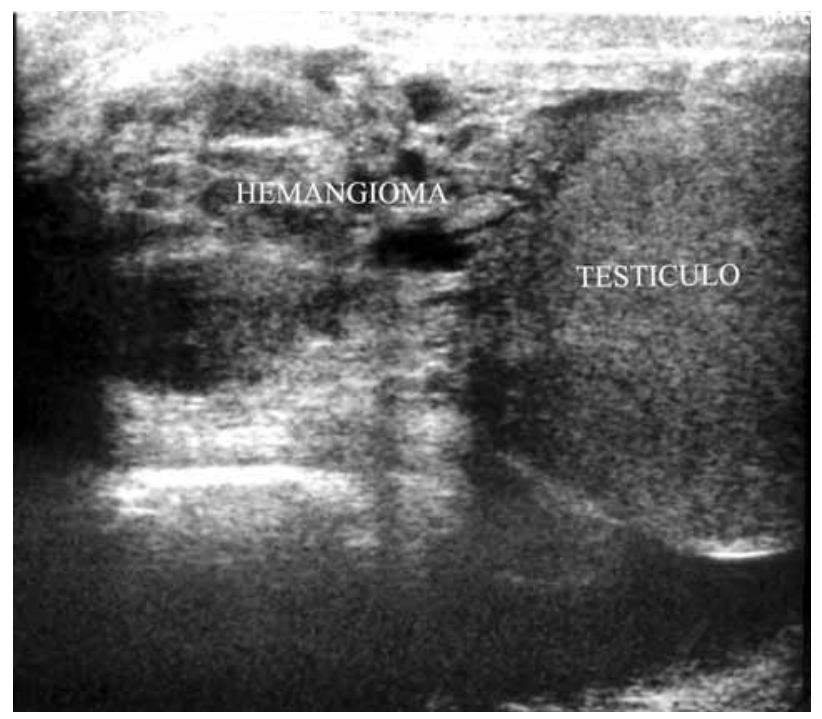

FIGURA 1

Dada la imagen ultrasonográfica que no admitía un diagnóstico seguro de benignidad, la edad del paciente y la afectación del epidídimo, se optó por realizar una orquiectomía radical derecha.

En el servicio de Patología de nuestro Complejo Hospitalario recibimos una pieza de orquiectomía que medía 8,5 × 5 × $3 \mathrm{~cm}$., mostrando una superficie externa lisa, brillante, sin signos infiltrativos. Al corte, observamos un hidrocele marcado, rodeando un parénquima testicular conservado, sin infiltración neoplásica. Sobre él y sin contacto con el mismo, surge una tumoración polimacroquística, a nivel de todo el epidídimo y cordón espermático con zonas pardo amarillentas irregularmente distribuidas y numerosos vasos con depósito de un material hemático coagulado y parcialmente organizado, rechazando por compresión al parénquima testicular. La tumoración peritesticular descrita tiene un eje mayor de 3,6 cm, y se correspondía con la imagen ecográfica. (Fig. 2)

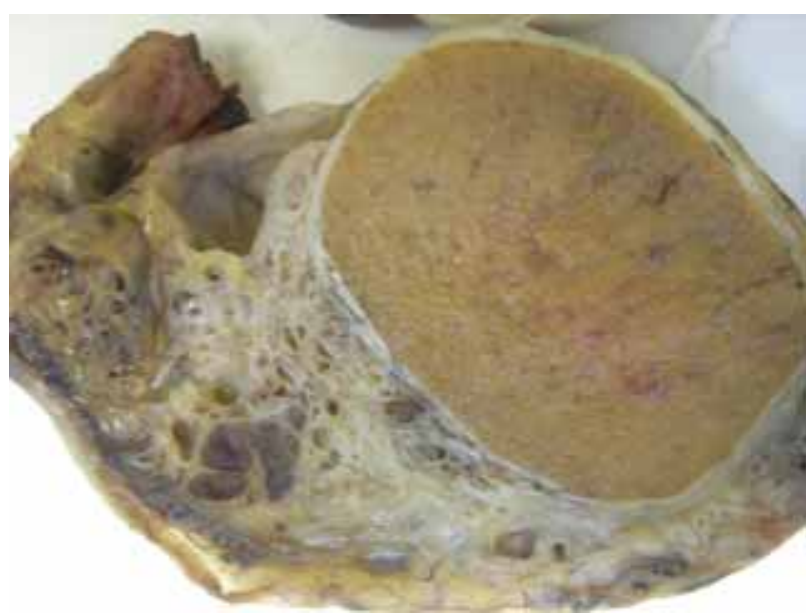

FIGURA 2

Microscópicamente, y junto a un parénquima testicular conservado, con las líneas espermatogénicas y espermatozoides propias de la edad, surge una banda fibrosa de separación tras la que encontramos -con conservación parcial del epidídimo- una neoformación vascular de luces grandes dilatadas entrecruzadas con un endotelio monoestratificado y de finas paredes, en el que las estructuras vasculares macro-microquísticas presentan algunas proyecciones digitiformes hacia la luz. Podemos observar en la seriación del área de conexión de la tumoración con la cabeza del epidídimo, el hecho de que aun surgiendo de su plexo vascular tiene conservación parcial de sus conductos (Figs. 3 y 4). Al mismo tiempo se observa que algunas luces tienen un material hemático organizado parcialmente como trombosis vascular (Fig. 5). Otras zonas intravasculares muestran depósito de histiocitos, celulas inflamatorias y material hemático en lisis, sin observar celularidad atípica.

El immunofenotipo tiene expresión positiva en los vasos para CD-34 y Factor VIII (Fig. 6).

Los vasos nutrientes epididimarios están en conexión con la neoformación vascular, la cual proviene histogenéticamente de los plexos pampiniformes. 


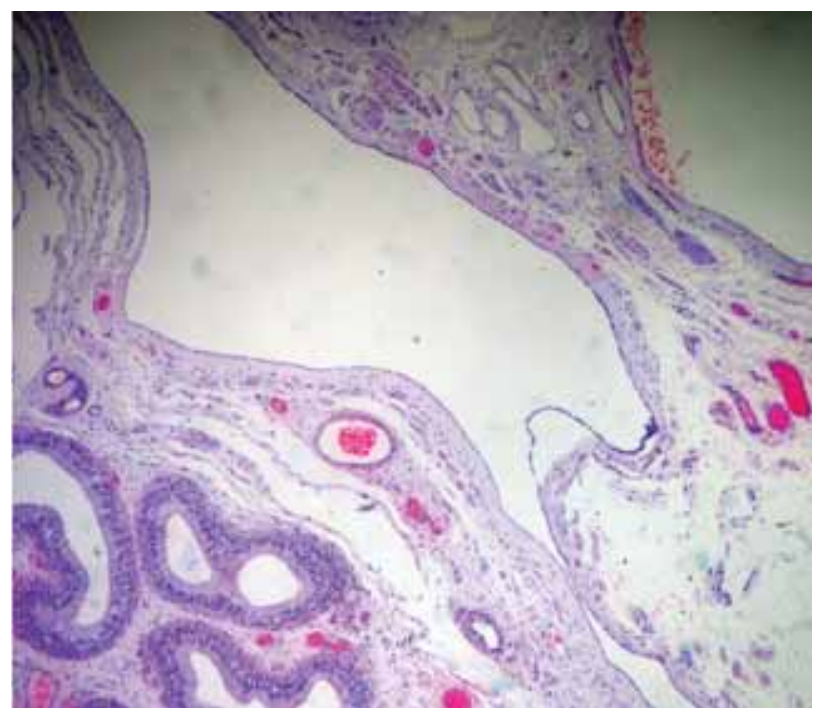

FIGURA 3

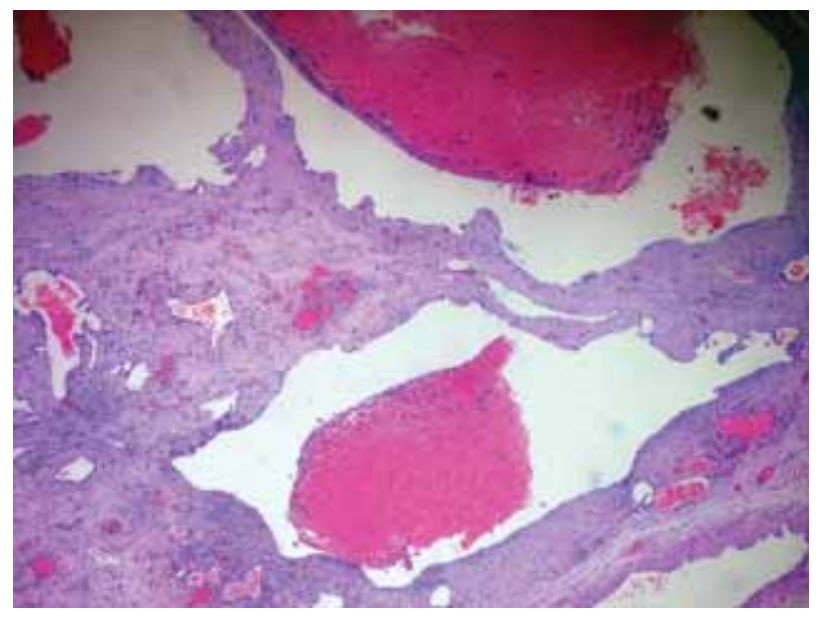

FIGURA 4

El diagnóstico se corresponde con un hemangioma cavernoso sinusoidal focalmente trombosado de epidídimo y con afectación parcial de cordón espermático. Hidrocele testicular.

\section{DISCUSIÓN}

Presentamos un caso singular de HI cavernoso de epidídimo. La rareza de su presentación no implica que se obvien estos tumores en el diagnóstico diferencial .

Los tumores paratesticulares, se presentan como masas escrotales (como en nuestro caso) o inguinoescrotales que pueden ser dolorosas, y en muchos casos acompañarse de hidrocele.

Desde el punto de vista anatomopatológico, es significativo el hecho de que existan muy pocos casos con contraste histológico en los casos halla-

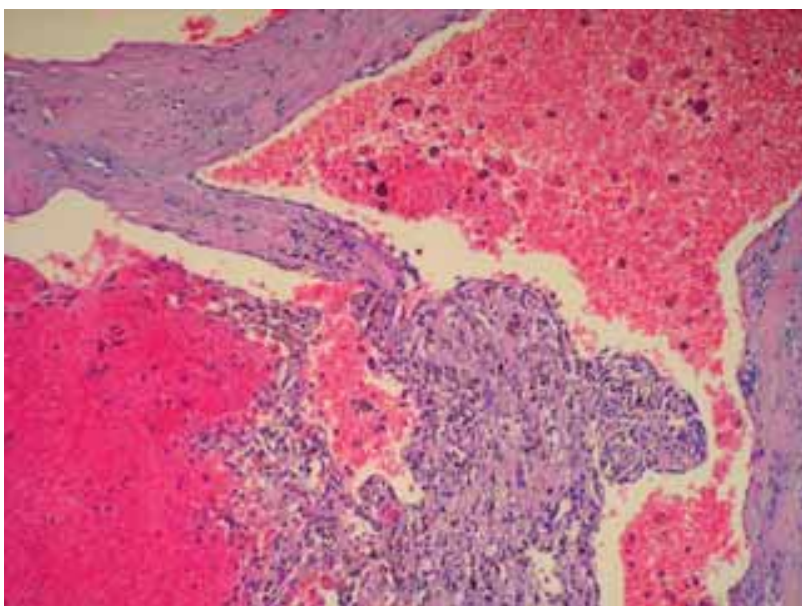

\section{FIGURA 5}

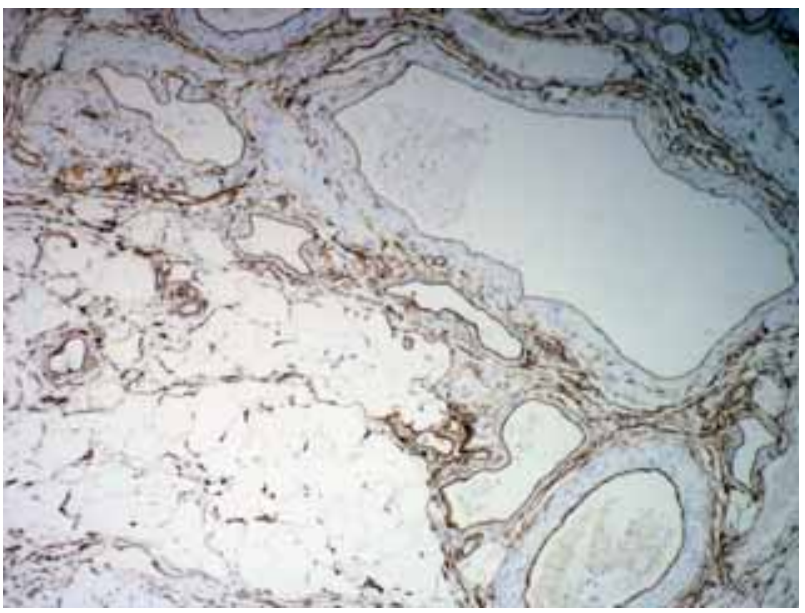

\section{FIGURA 6}

dos en la bibliografía internacional, lo que se presta a muchos interrogantes respecto del subtipo histológico.

El Diagnóstico Diferencial de los HI paratesticulares con otros tumores mesodérmicos benignos y malignos de la misma localización es, a veces, muy difícil, por confluir estirpes celulares muy semejantes y una ecogenicidad a veces confusa, más aún si el tumor se presenta con cambios "pseudoquísticos" (vasos dilatados) y con trombosis asociada ${ }^{8}$.

$\mathrm{Al}$ mismo tiempo, muchas publicaciones de HI son, en realidad, hemangiomas subcutáneos y no se ajustan al criterio de HI, el cual -para serlo- debe depender de los anejos testiculares y no de los cutáneos. Estos últimos son mucho más frecuentes y no tienen valor ni significación a la hora de estos estudios.

Es la ecografía el método de diagnóstico más utilizado en la evaluación inicial de las masas escrota- 
les, presentando dificultades en la mayoría de los casos y obligando en la casi totalidad de los procesos a una biopsia intraoperatoria, ya que incluso es muy difícil precisar preoperatoriamente, si se trata de un tumor benigno o maligno. Hemos indicado a su vez, el diagnóstico diferencial tan amplio que pueden presentar estas neoplasias antes de ser resecadas, y que solo se resuelve tras el estudio microscópico (Tabla 1). Ante una sospecha diagnóstica, la cirugía debe ser radical.

La baja incidencia de este tipo de neoplasias hace -además- que los tratamientos no estén sistematizados y se realicen terapias individualizadas, lo que condiciona dificultad en el estudio de los resultados, generando debates y controversias.

En la serie descrita por Mazal (22 casos) son los hemangiomas cavernosos los que muestran un intervalo etario mayor, en su presentación, desde los 15 a los 77 años, mientras que los hemangiomas histiocitoides y los capilares no alcanza ninguno los 50 años $^{37}$.

Como hallazgo único señalar que también se encontró -incluso- un hemangioma intraescrotal en un feto ${ }^{36}$ y con cierta, aunque inusual, mayor frecuencia, en testes no descendidos ${ }^{31}$.

Reflejan dolor en el momento del diagnóstico tan sólo el 31,5\% de los casos, sin poder valorar este hecho en los localizados en niños menores de 1 ó 2 años, como es evidente.

Hemos encontrado asociación con infartos testiculares en un reducido número de hemangiomas intraescrotales, pero no hemos datado ningún caso epididimario con trombosis vascular como en el que aquí recogemos.

\section{CONCLUSIONES}

La localización genital intraescrotal de los hemangiomas es excepcional. Hemos podido ver que los HI muestran tres grupos etarios de presentación: neonatos, primeras dos décadas de la vida y adultos mayores (a partir de 40-50 años).

Nuestro caso es el $4^{\circ}$ hemangioma cavernoso de epidídimo y el de mayor edad -al diagnóstico- descrito en la literatura, que se manifestó sin dolor por un engrosamiento del escroto, mal definido ecográficamente y que precisó de orquiectomía dada su afectación anexial testicular, a pesar de su benignidad histológica. No existían antecedentes de patología previa local o sistémica ni de síndromes asociados.
Los HI cavernosos son porcentualmente más frecuentes a nivel del parénquima testicular que en el resto de estructuras anexiales paratesticulares, aunque sin significación estadística.

No siempre el hidrocele -presente en nuestro caso- es signo de malignidad asociada. Tampoco el dolor es un signo patognomónico -biológico que acompañe a estos tumores vasculares, aunque la mayoría es por compromiso vascular: isquemia y necrosis. En nuestro caso, sin embargo, la trombosis generada en el HI no indujo patología dolorosa.

A diferencia de otros casos, en el nuestro, el parénquima testicular estaba conservado manteniendo incluso cierta filancia así como también la línea espermatogénica completa.

También y a pesar de los datos aportados por la bibliografía internacional, el número de $\mathrm{HI}$ hallados en adultos y niños, es muy semejante en incidencia, según hemos observado en la revisión realizada.

La trombosis y las calcificaciones, frecuentes en los hemangiomas cavernosos en otras localizaciones son poco frecuentes en los casos estudiados o en su defecto no constan en los artículos publicados.

El diagnóstico diferencial es muy amplio y en el caso de tumores del epidídimo, cordón espermático $\mathrm{y}$ anejos testiculares se plantean todas las posibilidades, tanto benignas como malignas, que dada la variabilidad de la ecografía, tan solo se resuelven intraoperatoriamente e incluso solo tras el estudio histológico reglado postorquiectomía.

Aunque el papel de la ecografía testicular ha cambiado en las últimas décadas debido a la utilización de altas frecuencias y el valor añadido que le ha dado la técnica de armónicos, sin embargo, en el caso de los HI, por su ecogenicidad en relación con su constitución tisular, precisa de un mayor espectro resolutivo dada su heterogeneidad ultrasonográfica.

Llama la atención que un gran número de publicaciones de HI se deban a autores japoneses, quedando sin resolver el interrogante de si realmente se debe a una mayor incidencia de estos tumores en Japón o bien a una tendencia alta de publicación de casos.

Por último, y en una época caracterizada por la comunicación, sería importante realizar una conferencia de consenso clínicopatológica, oncológica y radiológica para el estudio integral de los tumores escrotales, que no por su baja incidencia precisan menor atención que otras regiones anatómicas génitourinarias. 


\section{REFERENCIAS}

1. Khoubehi B, Mishra V, Ali M, Motiwala H, Karim O. Adult paratesticular tumours. BJU Internacional. 2002;90(7):707-715.

2. WHO-OMS Classification of tumours. Tumours of the urinary system and male genital organs. Eble J N, Sauter G, Epstein J I, Sesterhenn I A, Eds. IARC Press, Lyon (France). 2004 pp 273-276.

3. WHO-OMS Classification of tumours. Tumours of soft tissue and bone. Fletcher CDM, Unni K, Mertens F Eds. IARC Press, Lyon (France). 2002 pp 131-134

4. Kempson RL, Fletcher CDM, Evans H, Hendrickson MR, Sibley RK. Tumors of the soft tissues. Atlas of Tumor Pathology. Fascicle 30, 3th. series. Washington, DC 2001. pp 246-253.

5. Ulbright Th M, Amin MB, Young RH. Tumors of the testis, adnexa, spermatic cord and scrotum. Atlas of Tumor Pathology. Fascicle 25, 3th series. Washington, DC 1999 pp 352-353.

6. Robertson JW, Palitz S. Hemangioma of testis and epididymis. J Urol. 1954 ;72(5):908-910.

7. Boullay M. In discussion. Bull Soc Anat Paris 1851;26:194-197.

8. Sakamoto H, Saito K, Shichizyo T, Ishikawa K, Igarashi A, Yoshida H. Color Doppler ultrasonography as a routine clinical examination in male infertility. Int $J$ Urol. 2006;13(8):10731078.

9. Lozano V, Alonso P, Marcos-Robles J. Case report: sonographic appearance of cavernous haemangioma of the testis. Clin Radiol. 1994;49(4):284-285.

10. Espuela Orgaz R, Nogueras Gimeno MA, Passas Martinez J. Interés diagnóstico del ultrasonido en patología intraescrotal. Actas Urol Esp. 1983; 7:361-366.

11. Rodriguez Patrón R, Mayayo Dehesa T, Lennie Zuccarino A, Sanz Mayayo E, Arias-Fúnez F, García Navas R. Ecografía testicular. Arch Esp Urol 2006;59(4): 441-454.

12. Woodward PJ, Schwab CM, Sesterhenn IA. Extratesticular scrotal masses:radiologic-pathologic correlation. From the archives of the AFIP. Radiographics 2003;23:215-240.

13. Ruiz Liso JM, Ruiz García J, del Agua Arias C, Vaillo Vinagre A, Gutiérrez Martín A, García Pérez MA. Proliferación nodular y difusa fibrosa de la túnica vaginal del testículo. Rev Esp Patol. 2004;37(1):91-98.

14. Coindre JM. Immunohistochemistry in the diagnosis of soft tissue tumours. Histopathology. 2003;43:(1):1-16.

15. Goodwin WE. Multiple, bening fibrous tumors of tunica vaginalis testis. J Urol. 1946;56:438-447.

16. McClellan DS, Roscher A. Intrascrotal tumors in the older male. Int Surg. 1986;71(1):51-52.

17. Braeckman J, Van Tichelen G D. Unusual mass of the spermatic cord. J Urol. 2003;169(6):2298-2302.

18. Jayaram N, Ramaprasad AV, Chethan M, Sujay RP. Tumours and tumour-like conditions of the paratesticular region. A study of morphological features. Indian $\mathrm{J}$ Pathol Microbiol. 1998;41(3):287-295.

19. Weiss SW, Goldblum JR: Enzinger and Weiss. Soft Tissues Tumors. 4th edition. Mosby. 2001.

20. Miettinem M. Immunohistochemistry of solid tumors. Brief review of selected problems. APMIS. 1990;98(3):191-199.

21. Numakura K, Tsuchiya N, Inoue T, Yuasa T, Matsuura S, Satoh $\mathrm{S}$, et al. A case of testicular venous hemangioma. Hinyokika Kiyo. 2007;53(7):493-495

22. Takaoka E, Yamaguchi K, Tominaga T. Cavernous hemangioma of the testis: a case report and review of the literature. Hinyokika Kiyo. 2007;53(6):405-407.

23. Erdag G, Kwon EO, Lizza EF, Shevchuk M. Cavernous hemangioma of tunica albuginea testis manifesting as testicular pain. Urology. 2006;68(3):673-678.

24. Jeon YS, Cho SG, Kim WH, Choi SJ. Cavernous haemangioma of the spermatic cord in a child. Pediatr Radiol. 2006;36(12): 1323-1325.
25. Tan D, Kraybill W, Cheney RT, Khoury T. Retiform hemangioendothelioma: a case report and review of the literature. $\mathrm{J}$ Cutan Pathol. 2005;32(9):634-637.

26. Llarena Ibarguren R, Azurmendi Sastre V, Padilla Nieva J, Pertusa Peña C. Tumores no germinales de testículo. Arch Esp Urol. 2005;58(10):1031-1034.

27. Jira H, Hammoudi Y, Ferlicot S, Droupy S, Eschwege P, Benoit G, et al. Scrotal angiofibroblastoma. Prog Urol. 2004;14(4):561563.

28. Mitsuhashi M, Harima M, Kobayakawa H, Asakawa M. Racemose hemangioma of the scrotum: a case report. Hinyokika Kiyo. 2002;48(8):503-6.

29. Hirata H, Ohmi C, Takai K, Naito K. Intrascrotal hemangioma in a child: a case report. Hinyokika Kiyo. 2002;48(8):491-493.

30. Lin CY, Sun GH, Yu DS, Wu CJ, Chen HI, Chang SY. Intrascrotal hemangioma. Arch Androl. 2002;48(4):259-265.

31. Maeda T, Aoki M, Suzuki T. A case of arteriovenous hemangioma of the undescended testis. Hinyokika Kiyo. 2002;48(10): 611-613.

32. Hirata H, Ohmi C, Takai K, Naito K. Intrascrotal hemangioma in a child: a case report. Hinyokika Kiyo. 2002;48(8):491-493.

33. Elert A, Olbert P, Hegele A, Barth P, Hofmann R, Heidenreich A. Accuracy of frozen section examination of testicular tumors of uncertain origin. Eur Urol. 2002;41(3):290-293.

34. Tsolos C, Polychronidis A, Sivridis E, Kelidis G, Simopoulos C. Epithelioid hemangioendothelioma of the testis. J Urol. 2001;166(5):1834-1839.

35. Pan B, Song Y, Kong X, Xue Z, Guo Y. Primary epididymal tumors. Zhonghua Wai Ke Za Zhi. 1998;36(3):138-140.

36. Suriawinata A, Talerman A, Vapnek JM, Unger P. Hemangioma of the testis: report of unusual occurrences of cavernous hemangioma in a fetus and capillary hemangioma in an older man. Ann Diagn Pathol. 2001;5(2):80-83.

37. Mazal PR, Kratzik C, Kain R, Susani M. Capillary haemangioma of the testis. J Clin Pathol. 2000;53(8):641-642.

38. Gutierrez Minguez E, Serrano Bartolome JM, Claver Criado M.. Histiocytoid hemangioma of the epididymis. A very unusual entity. Actas Urol Esp. 2000;24(1):70-71.

39. Konya E, Uejima S, Ohnishi N, Sugiyama T, Kurita T. Venous hemangioma of the scrotum: a case report. Hinyokika Kiyo. 2000;46(10):731-733

40. Garcia Mediero JM, Alonso Dorrego JM, Nunez Mora C, Martinez-Pineiro Lorenzo L, Tejerina Gonzalez E, Nistal M, et al. Scrotal invasive angiomyofibroblastoma. First reported case. Arch Esp Urol. 2000;53(9):827-829.

41. Madrid Garcia FJ, Garcia S, Parra L, Rivas JA, Vesga Molina F, Gomez Cisneros S, et al. Hemangioma of the spermatic cord. Presentation of a case with review of the literature. Arch Esp Urol. 1998;51(5):499-502.

42. Pan B, Song Y, Kong X, Xue Z, Guo Y. Primary epididymal tumors. Zhonghua Wai Ke Za Zhi. 1998;36(3):138-140.

43. Awakura Y, Yamamoto M, Fukuzawa S, Fukuyama T. Cavernous hemangioma of the scrotum: a case report. Hinyokika Kiyo. 1998;44(10):751-753.

44. Yasui T, Akita H, Kobayashi K, Kohri K. Scrotal lymphangioma. Urol Int. 1998;61(3):178-180.

45. Blanco Gonzalez JE, Villo Sirerol N, Garcia Herrero MA, Alvarez-Coca Gonzalez J, Hontoria Briso-Montiano J, Santana Acosta A. Cavernous hemangioma of the scrotum. Arch Esp Urol. 1997;50(10): 1125-1127.

46. Pello Fonseca JM, Suarez Gonzalez JA, Mosquera Martinez MT, Rivas del Fresno M, Cuervo Calvo FJ, Muruamendiaraz Fernandez V. Intraparenchymatous vascular tumors of the testicle: report of a new case of cavernous hemangioma. Actas Urol Esp. 1997;21(10):998-1002.

47. Kolligian ME, Kogan SJ, Beneck D. Intrascrotal hemangioendothelioma in infancy. Urology. 1997;50(3):456-458. 
48. Yanai S, Tsutsumi H, Hotsubo T, Takahashi A, Miyao N, Satoh $\mathrm{M}$, et al. Development of a testicular haemangioma after interferon therapy for hepatic haemangiomas: a case report. Eur $\mathrm{J}$ Pediatr. 1997; 156(10):784-786.

49. Tomoyoshi T. Hemo-lymphangioma of the scrotum: a case report. Hinyokika Kiyo. 1997;43(4):307-309.

50. Koizumi S, Hayashida H, Hamaguchi A, Konishi T, Pak KI, Okada Y, et al. Hemo-lymphangioma of the scrotum: a case report. Hinyokika Kiyo. 1997;43(4):307-309.

51. Uchida K, Takahashi A, Miyao N, Takeda K, Tsutsumi H, Satoh M, et al. Juvenile hemangioma of the testis: analysis of expression of angiogenic factors. Urology. 1997;49(2):285-286.

52. Chen C, Cen H, Huang L. Primary epididymal tumors: report of 24 cases. Zhonghua Wai Ke Za Zhi. 1996;34(11):655-656.

53. Hidalgo F, Vinas F, Garcia JM, Urbina FO, Celis L, Abad M, Santos-Briz A. Capillary testicular hemangioma: a case report. Cir Pediatr. 1995;8(3):128-129.

54. Sendra Torres A, Picurelli Oltra L, Fernandez Rodriguez A, Alcala-Santaella Casanova C, Terradez Raro JJ. Testicular cavernous angioma: a difficult clinical diagnosis. Arch Esp Urol. 1995;48(2):203-205.

55. Ferrer FA, McKenna PH. Cavernous hemangioma of the scrotum: a rare benign genital tumor of childhood. J Urol 1995;153(4): 1262-1264

56. Kuraoka T, Taguchi K, Hosokawa S, Shimada K, Mori Y, Ikoma $\mathrm{F}$, et al. A case of capillary hemangioma of the testis in a child. Hinyokika Kiyo. 1994;40(4):361-363.

57. Calonje E, Fletcher CD, Wilson-Jones E, Rosai J. Retiform hemangioendothelioma. A distinctive form of low-grade angiosarcoma delineated in a series of 15 cases. Am J Surg Pathol. 1994;18(2):115-125

58. Mazzella FM, Sieber SC, Lopez V. Histiocytoid hemangioma of the testis. J Urol. 1995;153(3):743-744.

59. Chetty R. Epididymal cavernous haemangiomas. Histopathology. 1993;22(4):396-398.

60. Chetty R, Bandid S, Freedman D. Cavernous haemangioma of the epididymis mimicking a testicular malignancy. Aust N Z J Surg. 1993;63(3):235-237.

61. Allen PW, Ramakrishna B, MacCormac LB. The histiocytoid hemangiomas and other controversies. Pathol Annu. 1992;27 Pt 2:51-87.

62. Lopez Munoz A, Vilches Troya J, Aparicio Patino J, AlvarezOssorio JL, Castineiras Fernandez J. Hemangioma of the testis: a problem of clinical diagnosis. Arch Esp Urol. 1990;43(2): 192-194.

63. Banks ER, Mills SE. Histiocytoid (epithelioid) hemangioma of the testis. The so-called vascular variant of "adenomatoid tumor". Am J Surg Pathol. 1990;14(6):584-589.

64. Lozano JL, Menendez C, Adriazola M, Amo A, Tejeda E, Romero F. Cavernous angioma of the testis. Presentation of a new case.Actas Urol Esp. 1990;14(1):43-44

65. Tada M, Takemura S, Takimoto Y, Kishimoto T. A case of cavernous hemangioma of the testis. Hinyokika Kiyo. 1989;35(11): 1969-1971.

66. Pellice C Jr, Sabate M. Tumors of the spermatic cord. Preliminary report. Apropos of a hemangioma. Arch Esp Urol. 1989; 42(6):585-586

67. Komeda H, Horie M, Isogai K. A case report of scrotal hemangioma. Hinyokika Kiyo. 1989;35(5):911-912.

68. Aparicio Duque R, del Villar Negro A, Carpintero Sainz ML Sanchez Santos JA, Martinez-Sagarra Oceja JM, Santos Largo J. Cavernous angioma as a cause of ischemic testicular necrosis. Arch Esp Urol. 1987;40(5):353-354.

69. Chang SY, Ma CP, Tzeng CC. Benign testicular tumors. Eur Urol. 1987;13(4):242-245.

70. Ruesch R, Morger R. Cavernous hemangioma of the epididymis, a case report. Z Kinderchir. 1987;42(6):384-385.
71. Constancis P, Arvis G, Chamlou P, Conte J, Constancis E. Primary solid tumors of the epididymis. Apropos of 4 cases of benign tumor and one case of malignant mesothelioma. Ann Urol (Paris). 1987;21(6):387-390.

72. Ogawa O, Yoshimura N, Nishimura K, Nakagawa T, Nagata Y. A case of cavernous hemangioma of the testis. Hinyokika Kiyo. 1985;31(11):2060-2064.

73. Suarez-Ruiz JJ, Ramirez-Salcedo G, de la Torre-Rendon FE. Testicular infarction in puberty secondary to intratesticular hemangioma. Bol Med Hosp Infant Mex. 1985;42(12):771-774.

74. Gharpure KJ, Ahmed YB, Bhargava MK. Cavernous haemangioma of testis with acute testicular infarction a case report. Indian J Cancer. 1985 ;22(1):73-75.

75. Ducassou J, Richaud C, Hermanowicz M. A rare tumor of the scrotum. Apropos of one case of epididymal angioma. J Urol Nephrol (Paris). 1976;82(6):529-531.

76. Hargreaves HK, Scully RE, Richie JP. Benign hemangioendothelioma of the testis: case report with electron microscopic documentation and review of the literature.Am J Clin Pathol. 1982;77(5):637-642.

77. Fel P, Imreh A. Cavernous hemangioma of the testis. Orv Hetil. $1982 ; 123(14): 859-862$

78. Shental J, Fischelovitz J, Sudarsky M, Rizescu J. Hemangioma of the tunica albuginea testis. Eur Urol. 1982;8(6):370-371.

79. Fossum BD, Woods JC, Blight EM Jr. Cavernous hemangioma of testis causing acute testicular infarction. Urology. 1981;18(3):277-278.

80. Cricco CF Jr, Buck AS. Hemangioendothelioma of the testis: second reported case. J Urol. 1980;123(1):131-132.

81. Ishiguchi T, Sasaki T, Matsubara K, Kobayashi H, Kaii O, Mashita S, et al. Hemangioma of the scrotum-with special reference to angiographic and RI-angiographic findings. Rinsho Hoshasen. 1980;25(1):145-148.

82. Allen RG. Tumor masses of the neonate. Clin Perinatol. 1978;5 (1): 115-134

83. D'Esposito RF, Ferraro LR, Wogalter H. Hemangioma of the testis in an infant. J Urol. 1976;116(5):677-678.

84. Stein JJ. Hemangioendothelioma of the testis. J Urol. 1975; 113(2):201-203.

85. Pfitzenmaier NW, Wurster K, Kjelle-Schweigler M. Hemangioma of the Tunica albuginea testis. Urol Int. 1975;30(3):237-241.

86. Cooper TP, Anderson RG, Chapman WH. Hemangioma of the scrotum: a case report review and comparison with varicocele. J Urol. 1974; 112(5):623-626.

87. Scoppa F, Amitrano L. Testicular angioma. Clinical and histopathological study. Rass Int Clin Ter. 1972;52(3):163-171.

88. Schindera F. Successful intravenous prednisolone treatment of a cavernous hemangioma of the scrotum in an infant. Klin Padiatr. 1972;184(1):76-78.

89. Mininberg DT, Harley DP. Scrotal wall hemangioma in an infant. J Urol. 1971;106(5):789-790.

90. Dalla Pria AF, Vianello A. Angioma of the testis (case report). Riv Anat Patol Oncol. 1968;33:839-856.

91. Schwerdtner R. Fibromyoangioma of the epididymis. Z Urol. 1963;56:347-349.

92. Mahoney MT. Cavernous hemangioma of the scrotal septum. J Pediatr. 1956;49(6):744-745.

Correspondencia autor: Dr. J.M. Ruiz Liso

Servicio de Patología

Complejo Hospitalario de Soria. Hospital Virgen del Mirón

Carretera de Logroño, s/n - 42071 Soria.

Tel.: 975234 300/975 220850 (Ext. 3301)

E-mail autor: jruiz@hsor.sacyl.es

Información artículo: Original - Urología general

Trabajo recibido: octubre 2007

Trabajo aceptado: noviembre 2007 